\title{
Makes FLASH the difference between the intervention group and the treatment-as- usual group in an evaluation study of a structured education and treatment programme for flash glucose monitoring devices in people with diabetes on intensive insulin therapy: study protocol for a randomised controlled trial
}

Melanie Schipfer ${ }^{1 *}$ D, Carmen Albrecht ${ }^{1}$, Dominic Ehrmann ${ }^{1}$, Thomas Haak², Bernd Kulzer ${ }^{1}$ and Norbert Hermanns ${ }^{1}$

\begin{abstract}
Background: People with diabetes on intensive insulin therapy need sufficient glycaemic control to prevent the onset or progression of diabetic complications. The burden of multiple daily blood glucose self-testing can be lessened by novel diabetes technology like flash glucose monitoring systems which provide more information compared to self-monitoring of blood glucose. Despite this delivered additional information studies are showing no significant effect on $\mathrm{HbA}_{1 c}$ reduction, but a reduced time spent in a hypoglycaemic glucose range. We assume that users of these devices need additional education and training to integrate the delivered information into treatment decisions. Therefore, FLASH, an education and treatment programme, was developed. The programme evaluation follows herein.
\end{abstract}

Methods/design: Patients are recruited through 40 diabetes outpatient study centres located across Germany. They will be randomly assigned to participate in the education and treatment programme (intervention group) or to obtain treatment as usual (control group). All patients have to give blood samples and to answer a bench of questionnaires during baseline assessment, at the end of the intervention, and 6 months after the end of the intervention. Physicians will be asked to declare some additional clinical data (such as details of the diabetes therapy) for every patient at every one of the three assessment points.

Discussion: This study is conducted as a randomised controlled trial to test the hypothesis that the newly developed education and treatment programme combined with the use of a flash glucose monitoring device (intervention group) is superior to reduce $\mathrm{HbA}_{1 c}$ compared to the use of flash glucose monitoring alone (control group). The first results will be expected in 2018.

Trial registration: ClinicalTrials.gov, ID: NCT03175315. Registered on 2 May 2017.

Keywords: Diabetes, Flash glucose monitoring, Patient education and treatment programme, Psychoeducation, Self-management, Patient-reported outcome

\footnotetext{
* Correspondence: schipfer@fidam.de

${ }^{1}$ Research Institute Diabetes Academy Bad Mergentheim (FIDAM GmbH),

Johann-Hammer-Str. 24, 97980 Bad Mergentheim, Germany

Full list of author information is available at the end of the article
} 


\section{Background}

Diabetic patients on intensive insulin therapy with multiple daily injections or continuous, subcutaneous insulin infusion need to achieve sufficient glycaemic control to prevent the onset or progression of diabetic complications $[1,2]$, while at the same time avoiding the risk of severe hypoglycaemia.

Successful intensive insulin treatment requires close self-monitoring of blood glucose (SMBG). Measurement of blood glucose is especially important before main meals because the prandial insulin dose will vary with blood glucose level, planned carbohydrate consumption, and other factors (e.g. exercise, alcohol consumption). The multiple daily skin pricks and the need to carry a blood glucose meter, test strips, lancets, and blood glucose log books can be very trying, and patients often get frustrated [3]. However, novel technologies, such as flash glucose monitoring, can provide relief.

Flash glucose monitoring provides continuous measurement of interstitial glucose levels via a glucose sensor placed in the subcutaneous tissue of the upper arm $[4,5]$. This sensor can be left at the insertion site for 14 days, and the interstitial glucose level obtained whenever necessary by scanning with a reader or smartphone using near-field technology. In addition to the current glucose level, the reader or smartphone will also display arrows indicating the long-term trend in interstitial glucose level (slightly/ strongly increasing, slightly/strongly decreasing, or stable) and the course of the glucose level over the last $8 \mathrm{~h}$.

Flash glucose monitoring has several benefits over SMBG. Multiple daily skin pricks can be avoided. Glucose levels can be painlessly measured any number of times by scanning with a reader and, moreover, the results are automatically stored for 90 days. Software can display glucose patterns and provide information on additional parameters, such as time spent in predefined glycaemic ranges (e.g. hypoglycaemic or hyperglycaemic range), median and mean glucose values, or an estimation of the current glycated haemoglobin level $\left(\mathrm{HbA}_{1 \mathrm{c}}\right)$, based on the previous collected and stored interstitial glucose values.

This new technology also has some downsides. Users need to be educated on how to integrate all the additional information (e.g. trend arrows, previous course of glucose) into treatment decisions. Problematic glucose patterns requiring treatment adjustments can be easily identified, but only if the user has the necessary knowledge and skills.

Two previous studies $[1,2]$ have shown that flash glucose monitoring has no significant effect on $\mathrm{HbA}_{1 \mathrm{c}}$, although it does reduce time spent in the hypoglycaemic glucose range. We hypothesise that education on how to make optimal use of flash glucose monitoring devices would improve outcomes. We therefore developed a programme, FLASH, to support these patients. We are planning a randomised controlled trial (RCT) to determine the effect of FLASH on
$\mathrm{HbA}_{1 \mathrm{c}}$ as well as on secondary outcomes (i.e. time spent in different glucose ranges, patient-reported outcomes, and device satisfaction). The purpose of this paper is to present the research protocol of the planned study.

\section{Methods/design \\ Study setting}

The study is designed as a multicentre study. A total of 40 diabetes outpatient treatment centres located across Germany have been invited to recruit eligible patients for the study. A study centre is defined as a medical practice run by a diabetologist and where diabetes nurses or certificated diabetes educators (CDE) are also available. The course instructors for FLASH will be CDE. Prior to the study, the course instructors will undergo an intensive 8-h training in the study protocol and the education and treatment programme. This programme will be conducted by the research team. Course instructors will be provided with a written curriculum and audiovisual teaching material, along with detailed descriptions of each slide. Additionally, trainers from the coordination research institute (Research Institute of Diabetes Mergentheim: FIDAM) will visit each participating medical centre, and course instructors can then resolve any doubts about the conduct of the programme. In these visits FIDAM employees will confirm that the slides of the FLASH will operate and could be displayed in each medical praxis and that the course instructor knows how to conduct the programme, fully understands the content of the programme, can clarify any likely questions, and knows how to upload the therapy data from the flash glucose monitoring reader to Diasend ${ }^{\circ}$. During the course of study, all study centres will have a hotline to FIDAM for support. FIDAM will communicate with all participating study centres via newsletters where every new development can be presented promptly and reliably. Conversely, the coordinating centre will be available for the responsible personnel in the study centres for the entire duration of the study. In addition to conducting the education and treatment programme, the physicians at the study centres will be responsible for the therapy of their patients during and after the study.

\section{Study design}

This multicentre RCT will enroll diabetic patients on intensive insulin therapy who are using flash glucose monitoring. The patients will be randomised into one of two groups: an intervention group and a control group. Both groups will receive the flash monitoring device and technical instructions on its use; the intervention group will receive, in addition, FLASH training.

\section{Sample size calculation}

A total of 86 patients per group will be necessary to detect a mean difference of $0.3 \%$ and a standard deviation of 
$0.7 \%$ in $\mathrm{HbA}_{1 \mathrm{c}}$ reduction between the two treatment groups (Cohen's $d=0.43$ ), with an alpha error $=0.05$ and beta $=0.2$ (power $=0.8$ ). Given an expected non-evaluable rate of $20 \%$, a total of 216 participants will be needed, with 108 patients in each group.

\section{Recruitment and randomisation}

All participating study centres will identify eligible patients interested in participating in the study. The eligible patients will be offered an appointment to get to know the facts of the study, to clarify doubts, and to give signed informed consent. Because no education programme for patients using flash glucose monitoring devices is available, medical centres and patients are showing great interest in participating in this study. However, despite the great interest it is possible that the required sample size will not be achieved. In this case there are more medical centres available which can be contacted for patient recruitment. Patient recruitment will be stopped after 216 patients are enrolled.

Baseline assessment will take place over two visits. At the first visit, blood will be collected, baseline questionnaires will be completed, patients will be provided with the flash glucose monitoring device and receive technical instructions, and the sensor will be implanted. Blinded FIDAM staff will review all baseline assessments to confirm that the enrolled patients meet all the eligibility criteria. Then, randomisation of patients will be done centrally at FIDAM using SYSTAT version 12, with random assignment at the level of the study centre. The results will be sent to the respective study centres in sealed envelopes that will be opened only at the second baseline visit. At this second visit, 2 weeks after the first visit, patients will be informed of the group assignment. Glucose-level data from the reader will be uploaded via Diasend ${ }^{\oplus}$ (see below: 'Assessments').

\section{Inclusion and exclusion criteria Inclusion criteria}

Patients of either sex will be eligible for inclusion in the study if they fulfil the following conditions:

- Age 16-75 years

- On intensified insulin therapy/insulin pump therapy

- Previous participation in at least one structured diabetes education programme

- $\mathrm{HbA}_{1 \mathrm{c}}$ in the range of $7.5-14 \%$

- Reduction of $\mathrm{HbA}_{1 \mathrm{c}}$ as the therapeutic goal

- Able to understand, speak, and write German

- Willing to provide informed consent (if necessary, informed consent of the parents)

- Have an indication for using a flash glucose monitoring system
Indication for a flash glucose monitoring system will be decided by the treating physician. The decision will be based on the following: (1) frequent unexplicable glucose levels and need for multiple daily measurement of blood glucose; (2) severe hypoglycaemic events, especially during the night; hypoglycaemic unawareness; and (3) undue patient anxiety about the use of the lancet, but otherwise good compliance with the device.

\section{Exclusion criteria}

Patients will be excluded if they have any of the following:

- Diabetes duration $<1$ year

- Type 2 diabetes not on insulin, or on non-intensified insulin therapy

- Severe organic disease preventing regular participation in the training course

- Pregnancy

- Severe cognitive impairment

- Current treatment of a psychiatric disorder

- Renal disease requiring dialysis

The treating physician will confirm that each patient satisfies the inclusion and exclusion criteria by checking the health records or by clinical assessment. Once included in the study, patients may still be excluded from the analysis if they decide to leave the study at any point for any reason, or if they miss any one treatment appointment or one of the three assessment points.

The REPLACE and IMPACT studies [1, 2] were not able to show significant improvement in glycaemic control with flash glucose monitoring vs. SMBG. Assuming that education might be able to improve the efficacy of flash glucose monitoring, exclusion of patients with previous flash glucose monitoring system use is not necessary (since the device alone does not seem to be efficacious in improving the $\mathrm{HbA}_{1 \mathrm{c}}$ ). The main objective of this study is not to prove the efficacy of flash glucose monitoring systems, but rather to evaluate the efficacy of the FLASH education and treatment programme. However, if there are substantial differences between the control and intervention groups in previous flash glucose monitoring system use, adjustment will be made during the analysis for experience of using previous flash glucose monitoring systems.

\section{Content, structure and intervention of FLASH}

FLASH is a structured education and treatment programme that will be delivered in a group setting, with each group comprising three to eight patients in the age range 16-75 years. This wide age range was selected so that study centres could include as many eligible patients as possible. Previous experience has shown that it is difficult to recruit homogeneous age groups. Having patients of widely differing ages is 
not necessarily a disadvantage, since younger people with diabetes can learn from the experiences of older ones and vice versa. If important differences are observed between the different age groups, adjustment for these factors will be made during analysis of data.

FLASH aims to empower patients by providing information and teaching strategies for better self-management of diabetes. FLASH participants are encouraged to be actively involved in their treatment: they formulate their own therapy-related goals, learn to recognise patterns in their glucose levels and to identify trends, and also to use this information for optimising daily therapy decisions. During FLASH, participants are also invited to share their experiences with flash glucose monitoring with other group members. The ultimate objective is to teach patients to live active and normal lives through efficient use of flash glucose monitoring.

FLASH uses different modern educational techniques. The guiding principle is self-management, especially self-monitoring, self-assessment, and enhancing selftreatment. Participants are provided with written material and worksheets and are encouraged to test the contents of each lesson on their own and to then discuss their experiences in the group setting. They are introduced to computer-based data analysis software and learn how to use it for optimising their own therapy. Additionally, patients define what they personally hope to achieve by attending the FLASH programme. Course instructors are provided with core content about the interpretation of flash glucose monitoring results and also optional content in slides on a secondary level if more detailed information is needed on a certain topic (e.g. exercising or hypoglycaemia).

The FLASH programme takes place over 6 weeks. It consists of four sessions, lasting $90 \mathrm{~min}$ each. There is a 1week interval between sessions 1 and 2, and 2-week intervals between sessions 2 and 3 and sessions 3 and 4 . These intervals give patients time to practise the newly learned techniques in their daily lives. Table 1 provides an overview of FLASH.

The first session focusses on the function of the flash glucose monitoring system and on interpretation of the trend arrows. The emphasis will be on teaching patients how to make therapy adjustments based on the information derived from the trend arrows.

The second session introduces patients to ambulatory glucose profile (AGP) monitoring (modules 1-2 of the six modules on AGP). Patients will learn to systematically analyse their glucose profiles and to organise the information from the flash glucose monitoring system.

The third session focusses on pattern recognition (modules 3-6). Participants will learn how to interpret the AGP and use it for therapy adjustment. Simple case studies will be used to teach participants to recognise typical patterns. Possible causes for these patterns and the appropriate therapy adjustments will be discussed.

The fourth and final session focusses on the changes in the AGP due to therapy adjustments. Individual cases on therapy adjustment during FLASH will also be discussed. Participants will be given an opportunity to share practical tips on how to handle minor day-to-day problems.

FLASH was developed in consultation with a group of experts (diabetologists and diabetes educators with special experience in flash glucose monitoring) according to German and international guidelines. The contents and lessons were discussed with the experts over the course of 1 year and were refined based on patients' experiences with flash glucose monitoring. FLASH has a written curriculum to be followed by educators and study material for patients like worksheets used during courses as well as tasks for the time at home in which the patients will have to test the content of the preceding course.

Figure 1 shows how the patients were assigned to the two treatment arms.

\section{Outcome measures \\ Primary outcome}

The primary outcome will be change in glycaemic control analysed by estimating $\mathrm{HbA}_{1 \mathrm{c}}$. Testing will be performed in a central laboratory using the high-performance liquid chromatography (HPLC) method (normal range, 4.3-6.1\% or $23.5-43.2 \mathrm{mmol} / \mathrm{mol}$ ). Laboratory personnel will be blinded to the treatment allocation. All blood samples will be analysed once and then destroyed; specimens will not be stored for future use.

\section{Secondary outcomes}

Secondary outcomes will include medical and psychosocial measurements. These secondary outcomes will include: (1) time spent in different glycaemic ranges (hypo-/normo-/hyper-glycaemic status: $<70 \mathrm{mg} / \mathrm{dL} / 70$ $180 \mathrm{mg} / \mathrm{dL} />180 \mathrm{mg} / \mathrm{dL}$ ); variability of glucose level; (2) episodes of severe hypoglycaemia; (3) quality of life; (4) diabetes distress; (5) depression; (6) empowerment; and (7) satisfaction with the device and with the education.

\section{Assessments}

Patients will be assessed at three time points (Fig. 1):

1. At baseline ( $\mathrm{t} 0$ )

2. At the end of FLASH, after the fourth session (t1)

3. At follow-up 6 months after the end of FLASH (t2)

The first assessment will take place, as described above, over two visits with an interval of 2 weeks. The second assessment will occur immediately at the end of the FLASH programme or within 2 weeks after its end. At this visit the patients' glycaemic status and satisfaction with 
Table 1 Overview of FLASH: aims, content, and didactics

\begin{tabular}{|c|c|c|c|}
\hline Session & Aim & Key content & Special didactical feature \\
\hline \multirow[t]{2}{*}{1 First week } & \multirow{2}{*}{$\begin{array}{l}\text { Information about, and motivation for, } \\
\text { using of flash glucose monitoring }\end{array}$} & Principles of flash glucose monitoring & Clarification of features of the device \\
\hline & & Understanding trend arrows & Personal motivation \\
\hline \multirow[t]{2}{*}{2 Second week } & \multirow[t]{2}{*}{ Recognition of glucose pattern } & \multirow[t]{2}{*}{ Analysing glucose values and trends } & $\begin{array}{l}\text { Introduction to the } 6 \text { modules on AGP: } \\
\text { steps 1-2: understanding the AGP. }\end{array}$ \\
\hline & & & $\begin{array}{l}\text { Intensive discussion about glucose value } \\
\text { documentation and computer-based data } \\
\text { analysis software }\end{array}$ \\
\hline \multirow[t]{2}{*}{3 Fourth week } & \multirow[t]{2}{*}{$\begin{array}{l}\text { Therapy adjustment based on pattern } \\
\text { recognition and AGP }\end{array}$} & \multirow[t]{2}{*}{$\begin{array}{l}\text { Using data to recognise glucose } \\
\text { patterns and to adjust the therapy }\end{array}$} & $\begin{array}{l}\text { The } 6 \text { modules (continued): } \\
\text { modules 3-6: interpreting the AGP, and therapy } \\
\text { adjustment. }\end{array}$ \\
\hline & & & $\begin{array}{l}\text { Intensive discussion and amplification by personal } \\
\text { examples }\end{array}$ \\
\hline \multirow[t]{2}{*}{4 Sixth week } & \multirow[t]{2}{*}{ Check of the adjustment of the therapy } & \multirow[t]{2}{*}{ Dealing with barriers } & Reinforcement of lessons learned \\
\hline & & & Preparing methods for long-term management \\
\hline
\end{tabular}

AGP ambulatory glucose profile

the device will be assessed. Additionally, in the intervention group, satisfaction with the FLASH programme will also be evaluated. The third and last assessment will be at follow-up 6 months after the end of the FLASH programme. At this visit patients will answer follow-up questionnaires, give blood samples, and return the flash glucose monitoring device to the study personnel.

All assessments will be made by the personnel at the respective centre. All study centres are instructed by a personal visit and each study centre was offered a hotline and was audited by telephone. In addition, $50 \%$ of the study centres will be audited by FIDAM to check the quality of study conduct. Glycaemic data from the flash glucose monitoring system will be uploaded via
Diasend ${ }^{\circledR}$; thus, FIDAM can identify online incomplete data and notify the study centres whenever necessary.

Glucose values recorded through the flash glucose monitoring reader will be uploaded through the webbased diabetes management system Diasend ${ }^{\oplus}$, which will be installed on computers at each study centre. With individual patient codes, all flash glucose monitoring data will be uploaded by the responsible personnel at each study centre during the visits. Demographic data (age, gender, nationality, family status, housing situation, and educational level) and additional clinical data (weight and height, comorbidities, and details of the diabetes therapy) will be entered into Case Report Forms (CRFs) by the concerned physician. All CRFs will be in paper-and-pencil form; no electronic Case Report Forms

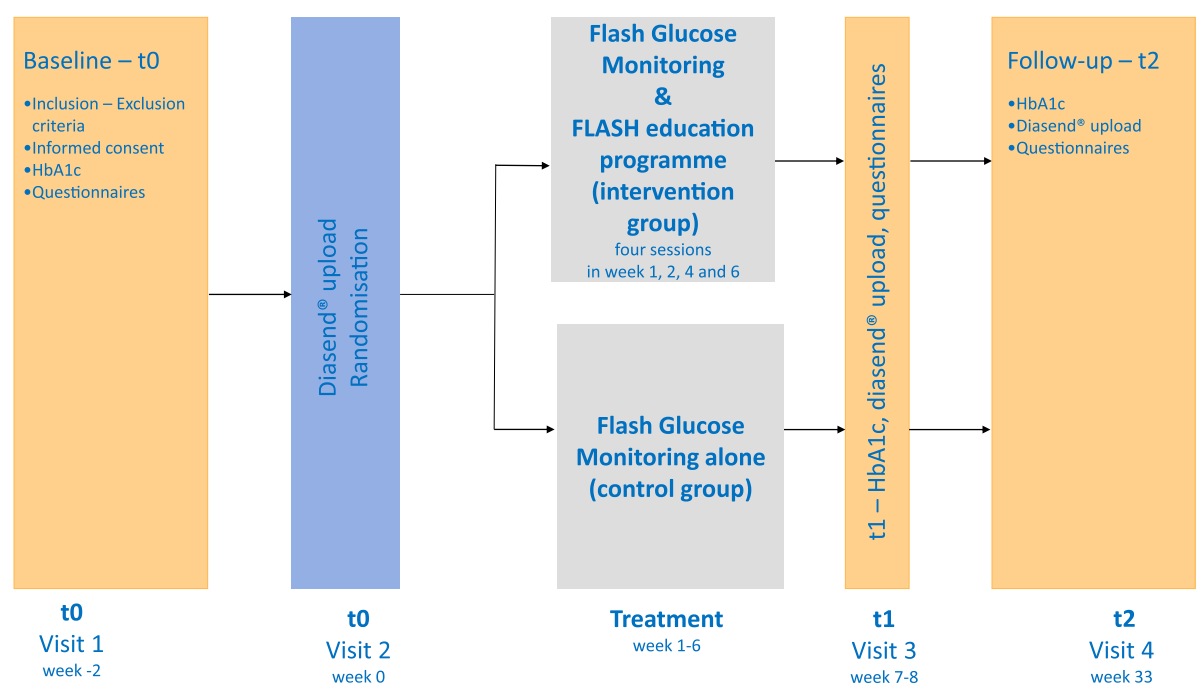

Fig. 1 The evaluation of FLASH - study flowchart 
(eCRFs) will be used. FIDAM will be responsible for the management of all collected data. This study is registered at ClinicalTrials.gov (identifier: NCT03175315) where all relevant information is available to the public. There will be no external safety committee since this is a non-pharmacological intervention. The incidence of serious adverse events and device-related adverse events will be collected via standardised reporting forms and reported to FIDAM.

Data on participants' emotional state and their experiences in living with diabetes will be collected through diabetes-, programme-, and device-related questionnaires:

- Diabetes Acceptance Scale (DAS) - to assess illness acceptance. Patients mark their intensity of agreement with 28 statements on a 4-point scale from 0 (not true) to 3 (totally true). A higher score indicates a higher level of acceptance [6]

- Diabetes Distress Scale (DDS) - a validated self-report scale to assess the current level of diabetes-related emotional distress. It has 28 items rated on a 6-point scale from 0 (no problem at all) to 5 (a very serious problem) [7]

- Problem Areas in Diabetes (PAID) Scale - a self-report form which builds up an index of emotional pressure, using 20 items rated on a 5 -point scale from 0 (not a problem) to 4 (serious problem) [8]

- General quality of life - the widely used World Health Organisation Well-being Index (WHO-5) self-report form to assess general quality of life. The scale consists of five questions, rated on a 6-point scale from 0 (at no time) to 5 (all the time). It is a measure of sense of well-being over the last 2 weeks [9]

- Health-oriented Quality of Life (EQ-5D; German version) - to assess the actual health status and to grade problems in five fields [10]

- Centre for Epidemiologic Studies Depression Scale (CES-D; German version) - to assess depression; it uses nine items rated on a 4-point scale from 0 (never/rarely) to 3 (often/always) [11]

- Personal Health Questionnaire (PHQ-8) - to assess mood issues over the last 2 weeks; eight items are rated on a scale that ranges from 0 (never) to 3 (nearly every day) [12]

- Hypoglycaemia Fear Survey (HFS) - to assess worries and behaviour related to hypoglycaemic events; it uses 18 statements related to troubles with hypoglycaemia at a variety of events; patients rate the troubles experienced on a 5-point scale that ranges from 0 (never) to 4 (always) [13]

- Diabetes Empowerment Scale - to assess empowerment and psychosocial self-efficacy; it uses 21 items to examine how difficulties in the daily routine are handled [14]

- Hypoglycaemia Awareness Scale (German version) - this consists of nine items. Scores range from 0 (maximum hypoglycaemic awareness) to 9 (minimum hypoglycaemic awareness) [15]

- Satisfaction with diabetes therapy - patients answer 10 questions on how satisfied they were with several aspects of their diabetes therapy over the last 4 weeks, with higher score indicating a higher level of dissatisfaction [16]

- Satisfaction with FLASH - the intervention group will be asked to evaluate FLASH. Participants will answer a total of 16 items

- Fidelity measure - in addition as a fidelity measure an 11-item scale will be applied. Participants of FLASH will be asked to what degree the educators addressed individual goals, how supported they felt in adjusting their diabetes therapy, and if the programme was presented in a structured way

- Glucose Monitoring Satisfaction Survey (GMSS) - patients will be asked to indicate their thoughts and emotions while using the flash glucose measurement system; there are 15 items that can be scored on a scale ranging from 1 (does not apply at all) to 5 (applies very strongly) [17]

- Use of technical possibilities - patients will be asked to describe their use of all the technical possibilities of the flash glucose monitoring system within the last 4 weeks; responses are graded on a scale ranging from 0 (not at all) to 4 (several times a day)

- Experiences with glucose measurement (Glucose Monitoring Assessment Tool; GMAT) - 19 statements are used to build up a score that reflects experiences related to measurement of glucose level; for example, 'the measurement of glucose is awkward for me' or 'I avoid measuring my glucose level if I have the feeling that the level is high'. [18]

Answering all the questionnaires will take about $30 \mathrm{~min}$ for each patient (the booster questionnaire administered at the end of the FLASH programme is slightly smaller). Figure 2 presents a summary of all the measurements.

Financial grants will be issued for patients who complete the study. These financial grants will not be given to the patients but to the study centres to compensate them for the additional effort (i.e. recruitment of patients, informing the patients about the study, obtaining informed consent, conduct of treatment, checking questionnaire data and electronic data for completeness, answering queries, and reporting serious or device-related adverse events). All educational materials will be supplied free to the study centres and patients. 


\begin{tabular}{|c|c|c|c|c|}
\hline Measurements & $\begin{array}{l}\text { Time } \\
\text { points }\end{array}$ & \begin{tabular}{|c} 
Baseline \\
(t0) \\
prior to \\
randomisati \\
on
\end{tabular} & \begin{tabular}{|} 
At the end of \\
the treatment \\
(t1)
\end{tabular} & $\begin{array}{l}\text { Follow-up } \\
\text { (t2) }\end{array}$ \\
\hline Inclusion/exclusion criteria $^{1}$ & & $\mathrm{X}^{1}$ & & \\
\hline Informed consent $^{1}$ & & $\mathrm{X}^{1}$ & & \\
\hline $\begin{array}{r}\text { Patient demography and medical } \\
\text { history }\end{array}$ & & $\mathrm{x}$ & & \\
\hline \multicolumn{5}{|l|}{ Primary outcome } \\
\hline $\mathrm{HbA}_{\mathrm{lc}}$ & & $\mathrm{x}$ & $\mathrm{x}$ & $\mathrm{x}$ \\
\hline \multicolumn{5}{|l|}{ Secondary outcomes } \\
\hline \multicolumn{5}{|l|}{ Glycaemic measures } \\
\hline Time in range & & $\mathrm{x}$ & $\mathrm{x}$ & $\mathrm{x}$ \\
\hline Variability of glucose level & & $\mathrm{x}$ & $\mathrm{x}$ & $\mathrm{x}$ \\
\hline Events of hypoglycaemia & & $\mathrm{x}$ & $\mathrm{x}$ & $\mathrm{x}$ \\
\hline Events of hyperglycaemia & & $\mathrm{x}$ & $\mathrm{x}$ & $\mathrm{x}$ \\
\hline $\begin{array}{l}\text { Psychological measures } \\
\text { (patient-reported outcomes) }\end{array}$ & $\begin{array}{l}\text { Number } \\
\text { of items }\end{array}$ & & & \\
\hline \multicolumn{5}{|l|}{ Diabetes related } \\
\hline Diabetes Acceptance Scale (DAS) & 28 & $\mathrm{x}$ & & $\mathrm{x}$ \\
\hline Diabetes Distress Scale (DDS) & 28 & $\mathrm{x}$ & $\mathrm{x}$ & $\mathrm{x}$ \\
\hline $\begin{array}{r}\text { Problem Areas In Diabetes Scale } \\
\text { (PAID) }\end{array}$ & 20 & $\mathrm{x}$ & & $\mathrm{x}$ \\
\hline $\begin{array}{r}\text { General Quality of Life } \\
\text { (WHO-5) }\end{array}$ & 5 & $\mathrm{x}$ & & $\mathrm{x}$ \\
\hline $\begin{array}{r}\text { Health-Oriented Quality of Life } \\
\text { (EQ-5D) }\end{array}$ & 5 & $\mathrm{x}$ & & $\mathrm{x}$ \\
\hline Depression Scale (CES-D) & 20 & $\mathrm{x}$ & & $\mathrm{x}$ \\
\hline $\begin{array}{r}\text { Personal Health Questionnaire } \\
\text { (PHQ-8) }\end{array}$ & 8 & $\mathrm{x}$ & $\mathrm{x}$ & $\mathrm{x}$ \\
\hline $\begin{array}{r}\text { Hypoglycaemic Fear Survey } \\
\text { (HFS) }\end{array}$ & 18 & $\mathrm{x}$ & & $\mathrm{x}$ \\
\hline \multicolumn{5}{|l|}{ Programme related } \\
\hline $\begin{array}{r}\text { Diabetes Empowerment Scale and } \\
\text { Self-Efficacy }\end{array}$ & 21 & $\mathrm{x}$ & $\mathrm{x}$ & $\mathrm{x}$ \\
\hline Hypoglycaemia Awareness Scale & 9 & $\mathrm{x}$ & & $\mathrm{x}$ \\
\hline Satisfaction with the diabetes therapy & 10 & $\mathrm{x}$ & $\mathrm{x}$ & $\mathrm{x}$ \\
\hline Satisfaction with the education ${ }^{2}$ & 16 & & $\mathrm{X}^{2}$ & \\
\hline Fidelity $^{2}$ & 11 & & $\mathrm{X}^{2}$ & \\
\hline \multicolumn{5}{|l|}{ Device related } \\
\hline $\begin{array}{r}\text { Glucose Monitoring Satisfaction } \\
\text { Survey (GMSS) }\end{array}$ & 15 & $\mathrm{x}$ & $\mathrm{x}$ & $\mathrm{x}$ \\
\hline Use of technical possibilities & 5 & $\mathrm{x}$ & $\mathrm{x}$ & $\mathrm{x}$ \\
\hline $\begin{array}{r}\begin{array}{r}\text { Glucose Monitoring Assessment Tool } \\
\text { (GMAT) }\end{array} \\
\end{array}$ & 19 & $\mathrm{x}$ & $\mathrm{x}$ & $\mathrm{x}$ \\
\hline \multicolumn{5}{|l|}{$\begin{array}{l}\text { '2 Weeks before baseline } \\
\text { 2Only for FLASH members }\end{array}$} \\
\hline $\begin{array}{l}\text { g. } 2 \text { Overview of all measur } \\
\text { sessment points }\end{array}$ & rement & the diff & erent & \\
\hline
\end{tabular}

\section{Planned data analysis}

Continuous data over all three time points will be summarised using descriptive statistics. Differences across the time points will be calculated for each group. The distributions of these differences will be compared using analysis of variance (ANOVA) for repeated measurements, with the FLASH group as the independent variable. If significant baseline differences are seen, the respective baseline values will be used as covariates.

Sometimes the questionnaire data may be skewed and may not fulfil the criteria for interval data. Assuming that at least rank-order data are available from the questionnaires, we will use either non-parametric tests for the evaluation of questionnaires and glucose data, or perform a transformation of the scores and glucose parameters into van der Waerden scores before applying parametric tests. Based on the data distribution and the scale of measurement, appropriate statistical methods will be selected. In the case of categorical data determined over several time points, we will use logistic regression analysis with adjustment for baseline values.

Statistical testing will be conducted at a significance level of $\alpha=0.05$. All analyses will be performed using SYSTAT version 12.0. For the intention-to-treat analysis missing data will be substituted by the last-observationcarried-forward method.

All data will be treated as confidential and be stored at FIDAM, the study coordination centre, for 10 years. Only FIDAM personnel will have access to the data during the study. FIDAM will monitor the flow of participants through each stage of the trial, and finally will be able to publish it.

\section{Discussion}

For quality of reporting, all standard protocol items recommended for international trials were taken in consideration according to the SPIRIT (Standard Protocol Items: Recommendations for Interventional Trials) Checklist, providing recommendations for a minimum set of ethical, scientific, and administrative elements which should be addressed in a clinical protocol [19] (see Additional file 1).

Patient enrolment will take place during the summer break and vacation periods and may be at different times at different centres.

The main results will be presented in conferences and reported in peer-reviewed publications. If FLASH is found to be beneficial the programme will be certified in Germany, so at the end every concerned patient will benefit.

\section{Trial status}

Protocol version number: 02.01.2018_1 (4 January 2018).

The study is currently (at the time of submission) recruiting participants. This can be seen at ClinicalTrials.gov (NCT03175315). Patient recruitment started on 2 May 2017, and will be completed by early October 2017. The first results are expected to be available in 2018 . 


\section{Additional file}

Additional file 1: SPIRIT 2013 Checklist: recommended items to address in a clinical trial protocol and related documents*. (PDF $168 \mathrm{~kb}$ )

\section{Abbreviations}

AGP: Ambulatory glucose profile; CDE: Certificated diabetes educators; CES-D: Centre for Epidemiologic Studies Depression Scale; CRF: Case Report Form; DAS: Diabetes Acceptance Scale; DDS: Diabetes Distress Scale; eCRF: Electronic Case Report Form; EQ-5D: Health-oriented Quality of Life - German version; FIDAM: Research Institute of Diabetes Mergentheim; FLASH: Name of the new patient education and treatment programme; GMAT: Glucose Monitoring Assessment Tool; GMSS: Glucose Monitoring Satisfaction Survey; HbA 1 c: Glycated haemoglobin A; HFS: Hypoglycaemia Fear Survey; HPLC: High-performance liquid chromatography; PAID: Problem Areas In Diabetes; PHQ-8: Personal Health Questionnaire; RCT: Randomised controlled trail; SMBG: Self-monitoring blood glucose; SPIRIT: Standard Protocol Items: Recommendations for Interventional Trials; SYSTAT: Statistical software, San Jose, CA, USA; WHO-5: World Health Organisation Well-being Index

\section{Acknowledgements}

We thank all study centres, patients, and others involved in this evaluation of FLASH.

\section{Funding}

The study was financially and materially funded by Abbott Diabetes Care, Germany. This support was an unrestricted grant and the funders had no role in the conceptualising of FLASH, data collection and analysis, preparation of the manuscript, or decision to publish.

Name and contact information of the trial sponsor:

FIDAM GmbH

Johann-Hammer-Str. 24

97980 Bad Mergentheim, Germany

\section{Availability of data and materials}

Data sharing is not applicable to this article as no datasets have been analysed. FLASH education materials are available from the corresponding author on request.

\section{Limitations}

We will note that financial grants could be a potential limitation of our study.

\section{Authors' contributions}

All authors (MS, CA, DE, TH, BK, and $\mathrm{NH}$ ) contributed substantially to the conception and design of the study, the acquisition and analysis of data, and the writing and revision of the manuscript. All have agreed to be accountable for the accuracy of the reported findings and the integrity of this work. All authors have given approval for publication of this paper. All authors read and approved the final manuscript.

\section{Ethics approval and consent to participate}

The study was approved by the Ethics Committee of the German Psychological Association (reference number NH032107). Ethical approval was received on 25 April 2017. All patients will have to provide signed informed consent before inclusion in the study. There are no plans for protocol modification.

\section{Consent for publication}

Not applicable.

\section{Competing interests}

The authors declare that they have competing interests.

MS received speakers' honoraria from Medtronic and Lilly Diabetes Germany. CA has no conflict of interest.

DE received speakers' honoraria from Berlin Chemie AG and Roche Diabetes Care.

$\mathrm{TH}$ is an advisory board member of Roche Diabetes Care, Sanofi, and Lilly He received speakers' honoraria from Berlin Chemie AG, Lilly, and AstraZeneca.

BK is an advisory board member of Berlin Chemie AG, Roche Diabetes Care Novo Nordisk, Medtronic, and Ascensia Diabetes Care. He received speakers' honoraria from Berlin Chemie AG, Novo Nordisk, Roche Diabetes Care, Abbott, Lilly, and Ascensia Diabetes Care. He has received grants in support of investigator trials from Berlin Chemie AG, Abbott, and Roche Diabetes Care.

$\mathrm{NH}$ is an advisory board member of Novo Nordisk, Abbott, Lilly, Roche Diabetes Care, and Ypsomed. He received speakers' honoraria from Novo Nordisk, Abbott, Berlin Chemie AG, Lilly, and Ypsomed. He has received grants in support of investigator trials from Dexcom, Berlin Chemie AG, Ypsomed, Abbott, and Roche Diabetes Care.

\section{Publisher's Note}

Springer Nature remains neutral with regard to jurisdictional claims in published maps and institutional affiliations.

\section{Author details}

${ }^{1}$ Research Institute Diabetes Academy Bad Mergentheim (FIDAM GmbH), Johann-Hammer-Str. 24, 97980 Bad Mergentheim, Germany. ${ }^{2}$ Diabetes Center Bad Mergentheim, Theodor-Klotzbücherstr. 12, 97980 Bad Mergentheim, Germany.

Received: 12 September 2017 Accepted: 18 January 2018

Published online: 05 February 2018

References

1. Bolinder J, Antuna R, Geelhoed-Duijvestijn P, Kröger J, Weitgasser R. Novel glucose-sensing technology and hypoglycemia in type 1 diabetes: a multicenter, on-masked, randomised controlled trial. Lancet. 2016;388(10057): 2254-63. https://doi.org/10.1016/S0140-6736(16)31535-5. Epub 2016 Sep 12.

2. Haak T, Hanaire H, Ajjan R, Hermanns N, Riveline JP, RAyman G. Flash Glucose-sensing technology as a replacement for blood glucose monitoring for the management of insulin-treated type 2 diabetes: a multicenter, openlabel randomized controlled trial. Diabetes Ther. 2017:8:55-73.

3. Polonski WH, Fisher L, Hessler D, Edelman SV. What is so tough about selfmonitoring of blood glucose? Perceived obstacles among patients with Type 2 diabetes. Diabet Med. 2014;31:40-6.

4. Hermanns N, Ehrmann D, Kulzer B. How much accuracy of interstitial glucose measurement is enough? Is there a need for new evidence? J Diabetes Sci Technol. 2016. https://doi.org/10.1177/1932296816677578.

5. Bailey T, Bode BW, Christiansen MP, Klaff LJ, Alva S. The performance and usability of a factory-calibrated flash glucose monitoring system. Diabetes Technol Ther. 2015;17(11):787-93.

6. Schmitt A, Reimer A, Ehrmann D, Kulzer B, Haak T, Hermanns N. Development and evaluation of a psychometric instrument to assess problems related to illness acceptance in diabetes. Diabetologia. 2015;58 Suppl 1:Abstract 946.

7. Polonsky WH, Fisher L, Earles J, Dudl RJ, Lees J, Mullan J, et al. Assessing psychosocial distress in diabetes: development of the Diabetes Distress Scale. Diabetes Care. 2005;28(3):626-31.

8. Polonsky WH, Anderson BJ, Lohrer PA, Welch G, Jacobson AM, Aponte JE, et al. Assessment of diabetes-related distress. Diabetes Care. 1995;18:754-60.

9. Brähler E, Muehlan H, Albani C, Schmidt S. Teststatistische Prüfung und Normierung der deutschen Versionen des EUROHIS-QOL-LebensqualitätsIndex und des WHO-5 Wohlbefinden-Index. Diagnostica. 2007;53:83-96.

10. Greiner W, Claes C. The EQ-5D of the EuroQoL Group. In: Schoeffski O, von der Schulenburg JM G, editors. Health economics evaluation. Heidelberg: Springer; 2007. p. 403-14.

11. Hautzinger M, Bailer J. German version of the Centre for Epidemiological Studies— Depression Scale. Göttingen: Hogrefe; 1993.

12. Kroenke K, Strine TW, Spitzer RL, Williams JB, Berry JT, Mokdad AH. The PHQ 8 as a measure of current depression in the general population. J Affect Disord. 2009;114(1-3):163-73.

13. Schipfer M, Ehrmann D, Hermanns N, Kulzer B, Haak T. Evaluation des Fragebogens zu Unterzuckerungen bei Erwachsenen mit Typ-1-Diabetes (HFS). Diabetologie und Stoffwechsel. 2016;11:130. https://doi.org/10.1055/s0036-1580877.

14. Anderson RM, Funnell MM, Fitzgerald JT, Marrero DG. The Diabetes Empowerment Scale: a measure of psychosocial self-efficacy. Diabetes Care. 2000;23:739-43

15. Clarke WL, Cox DJ, Gonder-Frederick LA, Julian D, Schlundt D, Polonsky W. Reduced awareness of hypoglycemia in adults with IDMM. A prospective study of hypoglycemic frequency and associated symptoms. Diabetes Care. 1995;18:517-22. 
16. Kulzer B, Bauer U, Hermanns N, Bergis KH. Entwicklung eines Problemfragebogens für Diabetiker zur Identifikation von Schwierigkeiten im Umgang mit der Krankheit. Verhaltenstherapie. 1995;5:A72.

17. Schipfer M, Ehrmann D, Haak T, Hermanns N, Kulzer B. Evaluation des Fragebogens zur Erfassung der Zufriedenheit des Glukosemonitorings bei Typ-1-Diabetes (T1-GMSS). Diabetologie und Stoffwechsel. 2016. https://doi. org/10.1055/s-0036-1580840.

18. Hermanns N, Ehrmann D, Schall S, Kulzer B, Haak T. Entwicklung und Evaluation eines Assessment-Tools zur Erfassung von Barrieren der Blutzuckerselbstkontrolle. DDG. 2015;10:315. https://doi.org/10.1055/s-00351549821.

19. Chan A-W, Tetzlaff JM, Altman DG, Laupacis A, Gøtzsche PC, Krleža-Jerić K, Hróbjartsson A, Mann H, Dickersin K, Berlin J, Doré C, Parulekar W,

Summerskill W, Groves T, Schulz K, Sox H, Rockhold FW, Rennie D, Moher D. SPIRIT 2013 Statement: defining standard protocol items for clinical trials. Ann Intern Med. 2013;158:200-7.

Submit your next manuscript to BioMed Central and we will help you at every step:

- We accept pre-submission inquiries

- Our selector tool helps you to find the most relevant journal

- We provide round the clock customer support

- Convenient online submission

- Thorough peer review

- Inclusion in PubMed and all major indexing services

- Maximum visibility for your research

Submit your manuscript at www.biomedcentral.com/submit
Biomed Central 